PROCEEDINGS OF THE

AMERICAN MATHEMATICAL SOCIETY

Volume 136, Number 9, September 2008, Pages 3205-3209

S 0002-9939(08)09317-9

Article electronically published on April 29, 2008

\title{
MAPS ON THE $n$-DIMENSIONAL SUBSPACES OF A HILBERT SPACE PRESERVING PRINCIPAL ANGLES
}

\author{
LAJOS MOLNÁR \\ (Communicated by N. Tomczak-Jaegermann)
}

\begin{abstract}
In a former paper we studied transformations on the set of all $n$-dimensional subspaces of a Hilbert space $H$ which preserve the principal angles. In the case when $\operatorname{dim} H \neq 2 n$, we could determine the general form of all such maps. The aim of this paper is to complete our result by considering the problem in the remaining case $\operatorname{dim} H=2 n$.
\end{abstract}

Wigner's famous theorem on the structure of quantum mechanical symmetry transformations plays a fundamental role in the probabilistic aspects of quantum mechanics. Mathematically, it can be formulated, among others, in the following way. If $H$ is a (real or complex) Hilbert space, then every bijective transformation on the set of all one-dimensional subspaces of $H$ which preserves the angle between subspaces is induced by a unitary or antiunitary operator on $H$. There is a natural one-to-one correspondence between the (closed) subspaces of $H$ and the orthogonal projections on $H$. Having this in mind, Wigner's theorem can also be stated as follows. If $P_{1}(H)$ denotes the set of all rank-one projections on $H$ and $\phi: P_{1}(H) \rightarrow$ $P_{1}(H)$ is a bijective map with the property that

$$
\operatorname{tr} \phi(P) \phi(Q)=\operatorname{tr} P Q \quad\left(P, Q \in P_{1}(H)\right),
$$

then there is a unitary or antiunitary operator $U$ on $H$ such that $\phi$ is of the form

$$
\phi(P)=U P U^{*} \quad\left(P \in P_{1}(H)\right) .
$$

In fact, the quantity tr $P Q$ is easily seen to equal the squared cosine of the angle between the ranges of $P$ and $Q$ as one-dimensional subspaces. In quantum mechanics, the quantity $\operatorname{tr} P Q$ is called the transition probability between the pure states represented by $P$ and $Q$.

As part of our work on Wigner's theorem and its generalizations (see 6], Sections 2.1-3 and, for further results, the last paragraph in the subsection 2.1.4 Remarks), in the paper [5] (also see [6, Section 2.1]) we considered the natural question to extend Wigner's theorem from the case of one-dimensional subspaces to the case of higher- (but fixed) dimensional subspaces. To do this, first we need a concept of angles between higher-dimensional subspaces. In [5] we used the notion of principal

Received by the editors March 28, 2007, and, in revised form, July 18, 2007.

2000 Mathematics Subject Classification. Primary 47B49, 15A30.

Key words and phrases. Wigner's theorem, projections, subspaces, principal angles, preservers.

The author was supported by the Hungarian National Foundation for Scientific Research (OTKA), Grant No. T046203, NK68040.

(C) 2008 American Mathematical Society Reverts to public domain 28 years from publication 
angles. The simplest way to define this concept is as follows. If $M, N$ are finitedimensional subspaces of $H$ and $P, Q$ are the corresponding orthogonal projections, then the principal angles between $M$ and $N$ are defined as the arccos' of the square rooted eigenvalues (counted according to multiplicity) of the positive finite rank operator $Q P Q$. The system of those numbers is denoted by $\angle(P, Q)$. This concept of principal angles was originally defined by Jordan [4 in 1875 in a geometrical way. Later it was proved to be probably the most useful notion of angles. For example, Hotelling [3] used it as a basic concept in his canonical correlation theory.

Let $H$ be an arbitrary (real or complex) Hilbert space and $n$ be a positive integer, $\operatorname{dim} H>n$. In [5] we obtained a Wigner-type result concerning the (not necessarily bijective) transformations of the set $P_{n}(H)$ of all rank- $n$ projections on $H$ which preserve the principal angles. In fact, in that paper we considered the cases when $n=1$ (this is just the original problem on rank-one projections) or $\operatorname{dim} H \neq 2 n$ and found that, for example, in the finite-dimensional case every such transformation is of the form $P \mapsto U P U^{*}$ with a unitary or antiunitary operator $U$ on $H$. The arguments in the proof were algebraic and based on some deep results concerning Jordan homomorphisms between rings. However, in [5] we could not handle the case when $n>1$ and $\operatorname{dim} H=2 n$. Because of the nice geometrical content of the problem we left this case as an open question. In fact, we formulated a conjecture. Observe that if $\operatorname{dim} H=2 n$, then the transformation $P \mapsto U(I-P) U^{*}(U$ is an arbitrary unitary or antiunitary operator) also maps $P_{n}(H)$ onto itself and preserves the principal angles. Indeed, as explained in 5, in the complex case this follows from [1, Exercise VII.1.11], while in the real case it was proved already by Jordan in [4] (also see [7, p. 310). This motivated us in [5] to formulate the conjecture that in the case under consideration our transformations are either of the form $P \mapsto U P U^{*}$ or of the form $P \mapsto U(I-P) U^{*}$. The aim of this paper is to verify the conjecture. Here we use an approach different from the one given in [5] as it is based on the use of commutativity preserving linear maps and not Jordan homomorphisms.

In what follows let $H$ be a finite-dimensional Hilbert space. We consider the real and complex case simultaneously. A unitary operator in the real case means an orthogonal transformation.

The main result of this paper reads as follows. We emphasize that our present approach using commutativity preserving maps instead of Jordan homomorphisms also allows us to recover the result in the formerly known finite-dimensional cases (with the only exception $n=1, \operatorname{dim} H=2$ ).

Theorem. Let $1<n \in \mathbb{N}$ and $H$ be a (real or complex) Hilbert space with dimension $\operatorname{dim} H>n$. Suppose that $\phi: P_{n}(H) \rightarrow P_{n}(H)$ is a transformation with the property that

$$
\angle(\phi(P), \phi(Q))=\angle(P, Q) \quad\left(P, Q \in P_{n}(H)\right) .
$$

If $\operatorname{dim} H \neq 2 n$, then there is either a unitary or an antiunitary operator $U$ on $H$ such that $\phi$ is of the form

$$
\phi(P)=U P U^{*} \quad\left(P \in P_{n}(H)\right) .
$$

If $\operatorname{dim} H=2 n$, then there is a unitary or an antiunitary operator $U$ on $H$ such that $\phi$ is either of the form

$$
\phi(P)=U P U^{*} \quad\left(P \in P_{n}(H)\right)
$$


or of the form

$$
\phi(P)=U(I-P) U^{*} \quad\left(P \in P_{n}(H)\right) .
$$

Remark that in the language of subspaces our result asserts that every map on the set of all $n$-dimensional subspaces of $H$ which preserves the principal angles is of the form $M \mapsto U(M)$ with some unitary or antiunitary operator $U$ if $\operatorname{dim} H \neq 2 n$, while it is either of the form $M \mapsto U(M)$ or of the form $M \mapsto U\left(M^{\perp}\right)$ if $\operatorname{dim} H=2 n$.

Before presenting the proof of the theorem we need some preparations. Denote $L_{s}(H)$ as the space of all self-adjoint linear operators on $H$ in the complex case or as the space of all symmetric linear operators on $H$ in the real case.

In what follows, we shall use the following useful notation. If $x, y \in H$, then $x \otimes y$ stands for the operator defined by

$$
(x \otimes y) z=\langle z, y\rangle x \quad(z \in H) .
$$

In the proof we shall also need the following auxiliary statements.

Lemma 1. Let $\mathcal{P}$ be any set of projections on $H$. If $\phi: \mathcal{P} \rightarrow \mathcal{P}$ is a transformation with the property that

$$
\operatorname{tr} \phi(P) \phi(Q)=\operatorname{tr} P Q \quad(P, Q \in \mathcal{P}),
$$

then $\phi$ has a unique real-linear extension onto the real-linear span $\operatorname{span}_{\mathbb{R}} \mathcal{P}$ of $\mathcal{P}$ and this extension is injective.

This is a part of Lemma 1 in [5].

Lemma 2. Let $1<n \in \mathbb{N}$. Then $\operatorname{span}_{\mathbb{R}} P_{n}(H)$ coincides with $L_{s}(H)$.

This is Lemma 4 in [5].

Lemma 3. Let $P, Q$ be projections on $H$. Then $Q P Q$ is a projection if and only if $P Q=Q P$.

This is a part of Lemma 5 in [5].

Now we are in a position to present the proof of our result.

Proof of the theorem. As $\phi: P_{n}(H) \rightarrow P_{n}(H)$ preserves the principal angles, for every $P, Q \in P_{n}(H)$ we obtain that the collection of all eigenvalues of $Q P Q$ (counted according to multiplicity) is equal to the collection of all eigenvalues of $\phi(Q) \phi(P) \phi(Q)$. This implies that $\operatorname{tr} Q P Q=\operatorname{tr} \phi(Q) \phi(P) \phi(Q)$ and hence we obtain

$$
\operatorname{tr} \phi(P) \phi(Q)=\operatorname{tr} P Q \quad\left(P, Q \in P_{n}(H)\right) .
$$

We apply Lemmas 1 and 2 to see that $\phi$ has a unique real-linear extension to $L_{s}(H)$ which is in fact injective. Denote this extension by the same symbol $\phi$. Hence, $\phi$ is a bijective linear transformation on $L_{s}(H)$.

The main step of the proof follows in which we show that $\phi$ preserves commutativity; i.e., it has the property that $\phi(A) \phi(B)=\phi(B) \phi(A)$ holds whenever we have $A B=B A$ for any $A, B \in L_{s}(H)$. To verify this, we first pick commuting projections $P, Q \in P_{n}(H)$. We know that the spectrum of $Q P Q$ equals the spectrum of $\phi(Q) \phi(P) \phi(Q)$. It follows that $Q P Q$ is a projection if and only if $\phi(Q) \phi(P) \phi(Q)$ is a projection. By Lemma 3 we can infer that $P$ and $Q$ commute if and only if $\phi(P)$ and $\phi(Q)$ commute. 
Now, pick two arbitrary commuting operators $A, B \in L_{s}(H)$. It follows from spectral theory that they are simultaneously diagonizable; i.e., there is an orthonormal basis $e_{1}, e_{2}, \ldots, e_{N}$ in $H$ such that $A, B$ can be written as

$$
A=\sum_{k=1}^{N} \alpha_{k} e_{k} \otimes e_{k} \quad \text { and } \quad B=\sum_{k=1}^{N} \beta_{k} e_{k} \otimes e_{k}
$$

with some scalars $\alpha_{k}, \beta_{k} \in \mathbb{R}$.

We next assert that there is a commuting family of projections in $P_{n}(H)$ whose real-linear span contains all of the rank-one projections $e_{k} \otimes e_{k}$. To see this, consider the set $\mathcal{P}$ of all rank- $n$ projections projecting onto the subspaces of $H$ linearly generated by any subset of $\left\{e_{1}, e_{2}, \ldots, e_{N}\right\}$ having exactly $n$ elements. Clearly, $\mathcal{P}$ is a commuting set of rank- $n$ projections. We verify that every rank-one operator $e_{k} \otimes e_{k}$ belongs to $\operatorname{span}_{\mathbb{R}} \mathcal{P}$ in a similar way as in the proof of [5, Lemma 4]. Obviously, without loss of generality we can consider, for example, $e_{1} \otimes e_{1}$. Let $E=e_{1} \otimes e_{1}+\ldots+e_{n+1} \otimes e_{n+1}$ and define

$$
P_{k}=E-e_{k} \otimes e_{k} \quad(k=1, \ldots, n+1) .
$$

Clearly, every $P_{k} \in \mathcal{P}$ can be represented by an $(n+1) \times(n+1)$ diagonal matrix whose diagonal entries are all 1's with the exception of the $k^{\text {th }}$ one which is 0 . The equation

$$
\lambda_{1} P_{1}+\ldots+\lambda_{n+1} P_{n+1}=e_{1} \otimes e_{1}
$$

gives rise to a system of linear equations with unknown scalars $\lambda_{1}, \ldots, \lambda_{n+1}$. The matrix of this system of equations is an $(n+1) \times(n+1)$ matrix whose diagonal consists of 0 's and its off-diagonal entries are all 1's. It is easy to see that this matrix is nonsingular, and hence $e_{1} \otimes e_{1}$ is a real-linear combination of $P_{1}, \ldots, P_{n+1}$. This proves our assertion that every $e_{k} \otimes e_{k}$ belongs to $\operatorname{span}_{\mathbb{R}} \mathcal{P}$, where $\mathcal{P}$ is a commuting subset of $P_{n}(H)$.

It now follows that $A$ and $B$ can be written as a real-linear combination of the same set of mutually commuting rank- $n$ projections. As $\phi$ is a real-linear transformation and it preserves commutativity between the elements of $P_{n}(H)$, we obtain that $\phi(A)$ and $\phi(B)$ commute.

We next apply a result of Choi, Jafarian and Radjavi [2] on commutativity preserving linear transformations which is well-known from the theory of linear preservers. This reads as follows. Let $H$ be a finite-dimensional real or complex Hilbert space with $\operatorname{dim} H \geq 3$. Let $\varphi: L_{s}(H) \rightarrow L_{s}(H)$ be a nonsingular linear transformation which preserves commutativity. If $H$ is real, then we have an orthogonal transformation $U$ on $H$, a scalar $c \in \mathbb{R}$ and a linear functional $f$ on $L_{s}(H)$ such that $\varphi$ is of the form

$$
\varphi(A)=c U A U^{*}+f(A) I \quad\left(A \in L_{s}(H)\right) .
$$

If $H$ is complex, then the same form is valid for $\varphi$ with the difference that $U$ is a either a unitary or an antiunitary operator on $H$.

Applying this result for $\phi$, we have a unitary or antiunitary operator $U$ on $H$, a scalar $c$, and a linear functional $f$ on $L_{s}(H)$ such that

$$
\phi(A)=c U A U^{*}+f(A) I \quad\left(A \in L_{s}(H)\right) .
$$

But our transformation $\phi$ maps $P_{n}(H)$ onto itself. Let us distinguish two cases. If $N \neq 2 n$, we easily obtain that $f(P)=0\left(P \in P_{n}(H)\right)$ and $c=1$. This gives us that $\phi$ is of the form (11). Suppose now that $N=2 n$. Then by $\phi\left(P_{n}(H)\right)=P_{n}(H)$ 
again, we obtain that for any $P \in P_{n}(H)$ we have either $f(P)=0$ which implies $c=1$ or $f(P)=1$ which implies $c=-1$. So, either $c=1$ or $c=-1$. In the former case we necessarily have $f(P)=0$ for every $P \in P_{n}(H)$, implying that $\phi$ is of the form (2), while in the case when $c=-1$ we necessarily have $f(P)=1$, which implies that

$$
\phi(P)=-U P U^{*}+I=U(I-P) U^{*} \quad\left(P \in P_{n}(H)\right) ;
$$

i.e., $\phi$ is of the form (3). The proof of the theorem is complete.

\section{REFERENCES}

1. R. Bhatia, Matrix Analysis, Springer-Verlag, 1997. MR1477662 (98i:15003)

2. M.D. Choi, A.A. Jafarian and H. Radjavi, Linear maps preserving commutativity, Linear Algebra Appl. 87 (1987), 227-241. MR878680 (88f:15003)

3. H. Hotelling, Relations between two sets of variates, Biometrika 28 (1935), 321-377.

4. C. Jordan, Essai sur la géométrie á n dimensions, Bull. Soc. Math. France 3 (1875), 103-174. MR 1503705

5. L. Molnár, Transformations on the set of all n-dimensional subspaces of a Hilbert space preserving principal angles, Commun. Math. Phys. 217 (2001), 409-421. MR.1821230 (2002b:81008)

6. L. Molnár, Selected Preserver Problems on Algebraic Structures of Linear Operators and on Function Spaces, Lecture Notes in Mathematics, Vol. 1895, Springer, 2006. MR2267033 (2007g:47056)

7. C.C. Paige and M. Wei, History and generality of the CS decomposition, Linear Algebra Appl. 208/209 (1994), 303-326. MR.1287355 (95f:15001)

Institute of Mathematics, P.O. Box 12, University of Debrecen, H-4010 Debrecen, HUNGARY

E-mail address: molnarl@math.klte.hu

URL: http://www.math.klte.hu/ molnarl/ 